\title{
Bundling Out Catheter-associated Urinary Tract Infection
}

\author{
Ramesh Venkataraman
}

Indian Journal of Critical Care Medicine (2020): 10.5005/jp-journals-10071-23493

Hospital-acquired infections (HAl) are the commonest cause of morbidity and mortality in hospitalized patients. ${ }^{1}$ The incidence of HAls may be higher in middle-income countries such as ours and the impact on cost of care greater. A large multicenter Indian study $^{2}$ reported that $12.2 \%$ of intensive care unit (ICU) patients developed an infection with an overall mortality of $28.4 \%$. In a recent large multicenter observational study of patients developing device-associated infections (DAI), 31.5\% had catheter-associated urinary tract infection (CAUTI). ${ }^{3}$ Shock requiring vasopressors was seen in $28.6 \%$ and reported hospital mortality was $11.9 \%$. Despite being a common DAl that adversely affects resource utilization and outcomes, CAUTI is amenable to easier prevention compared to other DAls. It is estimated that $17-69 \%$ of CAUTIs can be prevented if evidence-based infection control practices are strictly implemented. ${ }^{4}$

In the current issue, Charles et al. have conducted a beforeand after- intervention trial evaluating the impact of education and implementation of CAUTI prevention bundle. ${ }^{5}$ The authors have included a heterogeneous patient population comprising medical, surgical, neurotrauma, and cardiothoracic intensive care patients. During the preimplementation phase, routine standard catheter care was practiced and baseline data collected. During the implementation phase, healthcare workers (HCW) were educated in batches on recommended urinary catheter insertion and maintenance practices. Knowledge retention and comprehension were tested through objective structured clinical evaluation (OSCE). Meetings with all relevant intensive care units (ICUs) were held and availability of staff and resources regularized. During the post-implementation period, an infection control nurse audited the compliance with the bundle and conducted surveillance for incidence of CAUTI. With these measures, they have demonstrated decreased device utilization rates (0.7-0.56), decreased mean duration of catheter days (4.8-3.7), and a $60 \%$ reduction in CAUTI rates.

The study reinforces the fundamental idea that ensuring good infection control practices can make a huge impact on patient outcomes and morbidity. However, several important aspects of this study are noteworthy. Interventions such as infection control bundles do not logistically and ethically lend themselves to patient randomization and blinding, and hence the authors have used a pre- and post-implementation study design. Such a study design has several intrinsic limitations. Study period and therefore patient population evaluated could differ. The authors have, however, collected data meticulously, and patient characteristics remain similar between the two groups. Care processes change with time and could have contributed to improved catheter utilization and CAUTI rates. Factors such as volume and acuity of patients, experience and dedication of HCW performing insertion and maintenance of urinary catheter, and nurse patient ratio all can impact CAUTI rates independent of the intervention. It is well acknowledged that the simple act of observation can alter what is
Department of Critical Care Medicine, Apollo Hospitals, Chennai, Tamil Nadu, India

Corresponding Author: Ramesh Venkataraman, Department of Critical Care Medicine, Apollo Hospitals, Chennai, Tamil Nadu, India, Phone: +91 044 28296517, e-mail: ramesh@icuconsultants.com

How to cite this article: Venkataraman R. Bundling Out Catheterassociated Urinary Tract Infection. Indian J Crit Care Med 2020; 24(7):502-503.

Source of support: Nil

Conflict of interest: None

being observed-i.e., HCW would comply with CAUTI bundle once they knew they were audited. Random auditing at various times of the day negates this effect to some extent and the investigators have diligently performed that.

The investigators have combined several interventions into one: education and testing of HCW, resource planning, and allocation followed by strict auditing and feedback. The impact of each of this component cannot be assessed, and hence, all the benefit cannot be attributed only to the implementation of CAUTI bundle. Traditionally, education of HCWs has not been an effective tool in changing clinical practices. Commonly, noncompliance with evidence-based infection control practices is due to lack of human and material resources. It is likely that meeting with respective ICU leaders, understanding care processes specific to their ICU, and human and material resource planning and allocation could have impacted outcomes much more than the CAUTI prevention bundle itself. The overall incidence of CAUTI at baseline in this study is very high $(10.7 / 1,000$ catheter days) that any care process improvement is likely to decrease the rates. The very high initial rates likely explain the $60 \%$ reduction in CAUTI rates. Although patients from several ICUs were enrolled, majority of patients in this study have neurological or cardiac disease, and it is in these patients that urinary catheters are generally left longer due to inability to void or for strict monitoring of intake and outputs. This patient population hence could be more susceptible to CAUTIs, and results cannot be extrapolated to general medical/surgical patients with lower baseline risk of CAUTI.

Despite the abovementioned limitations, this study is valuable in providing us several important lessons. The investigators have demonstrated that a consorted system-centered multidisciplinary approach is possible and effective in decreasing CAUTI. First, it is commendable that they first could get buy-in from all the relevant stakeholders by individually meeting them to create awareness and understand the care processes and any lacunae that could be sealed. Second, they have involved HCW at all levels, educated them, ensured knowledge transfer, motivated them, and rewarded them for performance. Third, they have addressed issues related to the system and resource availability and have restructured them

(c) The Author(s). 2020 Open Access This article is distributed under the terms of the Creative Commons Attribution 4.0 International License (https://creativecommons. org/licenses/by-nc/4.0/), which permits unrestricted use, distribution, and non-commercial reproduction in any medium, provided you give appropriate credit to the original author(s) and the source, provide a link to the Creative Commons license, and indicate if changes were made. The Creative Commons Public Domain Dedication waiver (http://creativecommons.org/publicdomain/zero/1.0/) applies to the data made available in this article, unless otherwise stated. 
to individually suit their needs. Last, creating a checklist of items enhances uniformity and paves for easy audit. Implementing strict indications to insert a urinary catheter and its removal has decreased their catheter utilization rates and number of catheter days both of which have significantly contributed to their decreased CAUTI rates. The steps undertaken by the investigators are practical and can be adapted into any ICU to minimize CAUTI rates. The study highlights the mandatory need for every ICU to invest in a dedicated surveillance mechanism to monitor compliance with infection control practices and audit rates of infections routinely and provide timely feedback to practicing clinicians. This crucial step will decrease device-associated infections and related morbidity and mortality enormously.

\section{References}

1. Rosenthal VD, Maki DG, Salomao R, Moreno CA, Mehta Y, Higuera F, et al. Device-associated nosocomial infections in 55 intensive care units of 8 developing countries. Ann Intern Med 2006;145(8):582-591. DOI: 10.7326/0003-4819-145-8-200610170-00007.

2. Divatia JV, Amin PR, Ramakrishnan N, Kapadia FN, Todi S, Sahu S, et al. Intensive care in india: The indian intensive care case mix and Practice patterns study. Indian J Crit Care Med 2016;20(4):216-225. DOI: 10.4103/0972-5229.180042.

3. Venkataraman R, Divatia J, Ramakrishnan N, Chawla R, Amin P, Gopal $P$, et al. Multicenter observational study to evaluate epidemiology and resistance patterns of common intensive care unit-infections. Indian J Crit Care Med 2018;22(1):20-26. DOI: 10.4103/ijccm. IJCCM_394_17.

4. Safety P. National Healthcare Safety Network (NHSN) Overview. [cited 2017 Jul 31]; Available from: https://www.cdc.gov/nhsn/PDFs/ pscManual/1PSC_OverviewCurrent.pdf.

5. Soundaram GVG, Sundaramurthy R, Jeyashree K, Ganesan V, Arunagiri $\mathrm{R}$, Charles J. Impact of care bundle implementation on incidence of catheter associated urinary tract infection: a comparative study in the intensive care units of a tertiary care teaching hospital in south India. Indian J Crit Care Med 2020;24(7):544-550. 